\title{
Coping with COVID-19 stress: Associations with depression and anxiety in a diverse sample of U.S. adults
}

\author{
Mary Jo Coiro ${ }^{1}$ - Kelly H. Watson ${ }^{2}$ - Abby Ciriegio ${ }^{2} \cdot$ Maile Jones $^{2} \cdot$ Amy R. Wolfson $^{1}$. Jennie Reisman ${ }^{2}$. \\ Bruce E. Compas ${ }^{2}$
}

Accepted: 28 October 2021

(c) The Author(s), under exclusive licence to Springer Science+Business Media, LLC, part of Springer Nature 2021

\begin{abstract}
The COVID-19 pandemic has brought unprecedented levels of stress to individuals in the U.S. and throughout the world. These high stress levels place individuals at risk for symptoms of anxiety, depression, and other psychiatric disorders. The current study applies a control-based model of coping to contribute to the development of evidence-based interventions to promote resilience. Data were collected online from April 22 through July 12, 2020. Data from two samples of U. S. community adults who completed an online battery of standardized questionnaires were combined $(\mathrm{N}=709)$. More than a quarter reported moderate to severe levels of depression symptoms, and more than one-fifth reported moderate to severe levels of anxiety symptoms; symptom levels were higher among adults who reported more COVID-19-related stress. As hypothesized, multiple regression analyses indicated that greater use of primary and secondary control coping was associated with lower symptom levels, whereas greater use of disengagement coping was associated with higher symptom levels, above and beyond the association of stress with symptoms. Race and ethnicity emerged as important moderators of these associations, indicating that what constitutes adaptive coping varies according to characteristics of the individual. Implications for public health policy and clinical practice are discussed.
\end{abstract}

Keywords COVID-19 $\cdot$ Stress $\cdot$ Coping $\cdot$ Mental health $\cdot$ Racial/ethnic diversity

The global pandemic of the 2019 novel coronavirus disease (COVID-19) has presented an international public health crisis of a magnitude not seen in over a century. As of November 2021, over 200 million cases and more than 5 million deaths were documented worldwide (World Health Organization [WHO], 2021). Demands on public health have been far reaching, most dramatically in the overwhelming need for accurate and rapid testing for the virus and resources in hospital intensive care units to treat acutely ill patients.

In addition to needing effective methods to prevent and treat COVID-19, the pandemic has brought unprecedented stress to individuals in the U.S. and throughout the world. Stressors related to the pandemic include direct effects of the virus, such as being diagnosed with and treated for the disease; and indirect effects such as job loss or income

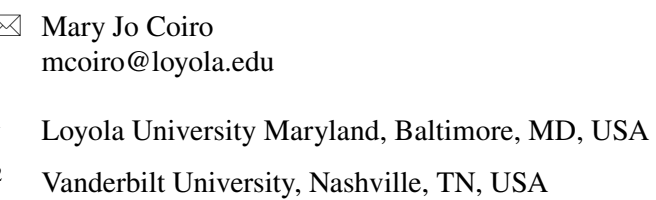

disruptions, closures of schools and agencies that provide essential care and support to families, and social isolation, interpersonal conflict, and loneliness as a result of complying with social distancing guidelines (e.g., Ahuja, 2021; Kujawa et al., 2020; Taylor et al., 2020). These COVID19-related stressors place individuals at risk for symptoms of depression, anxiety, and other psychiatric disorders. Furthermore, in the U.S. these stressors have coincided with a period of heightened racial tension and a contentious political climate.

Given these significant stressors, evidence-based guidance and resources on effective ways to cope with the myriad of COVID-19 related stressors are a public health priority (Gruber et al., 2020). Existing resources are limited in at least two ways: (1) information reflects generic suggestions for coping with stress (e.g., importance of sleep, regulated breathing, limiting alcohol intake) rather than with the unique stressors associated with COVID-19; and (2) there is no evidence on how COVID-19 sources of stress and effective ways of coping with these stressors may differ as a function of race, ethnicity, and other individual 
difference factors. The current study addressed these important research gaps to provide the basis for evidence-based resources to enhance resilience in adults during the COVID19 pandemic.

\section{COVID-19 Stress, Coping, and Mental Health Problems}

The COVID-19 pandemic has triggered an increase in symptoms of several psychiatric disorders, particularly depression and anxiety (Gao et al., 2020; Xiong et al., 2020). For example, among a representative sample of U.S. adults in June 2020, 40.9\% reported at least one adverse mental or behavioral health condition related to the pandemic, including symptoms of anxiety or depression (30.9\%), symptoms of a trauma- or stressor-related disorder (26.3\%), new or increased substance use (13.3\%), and serious thoughts about suicide (10.7\%; Czeisler et al., 2020).

Public health models of response to the pandemic have highlighted the importance of enhancing skills for coping with stress as part of building resilience and preventing mental health problems (e.g., Gruber et al., 2020; Kaslow et al., 2020; Ogueji et al., 2021; Wang et al., 2021). The selection of coping strategies may be affected by stressors located on the micro (e.g., race/ethnicity or sex), mezzo (e.g., social support or employment context), and macro (e.g., state or federal social distancing policies) level (Wang et al., 2021). Researchers are examining the ways that individuals are coping with COVID-19 stress and the mental health correlates of subtypes of coping (e.g., de Bruin, 2020; Novacek et al., 2020; Shamblaw et al., 2021; Shanahan et al., 2020; Shechter et al., 2020). For example, behavioral strategies such as keeping a daily routine, regular physical activity, engaging in religious/spiritual practices, forms of problem-focused coping (e.g., seeking advice from experts, talking to others), and cognitive strategies such as positive reappraisal/reframing have been associated with lower levels of distress (e.g., Gerhold, 2020; Shamblaw et al., 2021; Shanahan et al., 2020). In an on-line longitudinal study of 395 community adults, avoidance coping strategies including self-blame were associated with increased anxiety symptoms over a one-month period (Shamblaw et al., 2021). However, research to date can be built upon in several ways. First, there is an absence of theory-driven research and it is unclear what conceptual models have guided the choice of measures and the conceptualization of coping. Second, measures of coping have typically been relatively brief and may not capture the full range of strategies that may be relevant to successful adaption to the pandemic (e.g., Gerhold, 2020; Shamblaw et al., 2021; Shechter et al., 2020). Third, measures used in some studies have lacked a clear factor structure to organize the subtypes of coping (e.g.,
Asmundson et al., 2020; Ogueji et al., 2021; Shanahan et al., 2020). Taken together, these concerns highlight the need for research informed by models of coping using comprehensive and well-validated measures.

\section{Control-Based Model of Coping}

The control-based model of coping offers a framework for measuring and understanding ways of responding to situation-specific stressors that can provide an evidence base for the development of interventions to promote resilience during the pandemic (Compas et al., 2017; Weisz et al., 1994). The control-based model includes three coping domains: primary control coping (e.g., problem-solving, emotional modulation), secondary control coping (e.g., acceptance, cognitive reappraisal), and disengagement coping (e.g., avoidance, denial). In a comprehensive meta-analysis, the strongest associations of various subtypes of coping were found for primary and secondary control coping with internalizing and externalizing symptoms of psychopathology in children and adolescents (Compas et al., 2017). This model has generated extensive research to identify adaptive and maladaptive ways of coping with controllable and uncontrollable stressors ranging from acute and chronic illness, economic strain, war, and parental mental illness (e.g., Compas et al., 2017). Additionally, this model has been used in the development and testing of cognitive-behavioral interventions to improve coping skills and enhance resilience in adolescents and families (e.g., Bearman \& Weisz, 2009). Further, as reflected in the "goodness-of-fit" hypothesis, the efficacy of these different types of coping is hypothesized to differ as a function of the degree of actual and perceived control over specific sources of stress. For example, primary control coping efforts are associated with lower symptoms of anxiety and depression when used in response to controllable versus uncontrollable stressors (e.g., Finkelstein-Fox \& Park, 2019), whereas secondary control strategies are associated with lower distress in response to uncontrollable versus controllable stressors (Tu et al., 2016).

The control-based conceptual model is well suited for the identification of effective ways to cope with the COVID-19 pandemic for several reasons. First, the COVID-19 global pandemic is characterized by both controllable (e.g., separation from friends and family) and uncontrollable (e.g., being laid off, being hospitalized) stressors. Therefore, both primary control and secondary control coping may be important in managing these stressors. Second, coping is viewed as responsive to the demands of specific stressors and therefore can capture responses to the wide range of stressors presented by the COVID19 pandemic. The authors are unaware of published research using the control-based model in the context of 
this pandemic. Third, the model has been applied successfully in previous research to assess coping and stress reactivity in adults in response to two of the most salient aspects of the global COVID-19 pandemic-coping with medical illness (e.g., Compas et al., 1999a, 1999b; Compas et al., 2015) and economic hardship and strain (e.g., Wadsworth \& Compas, 2002). Fourth, the factor structure of the control-based model of coping has been validated using confirmatory factor analysis across a wide range of diverse populations in regard to age, race, ethnicity, and type of stress (Compas et al., 2017).

\section{Potential Moderators of Coping and Psychological Distress}

Individual differences may also moderate associations between coping and distress. Sociodemographic factors are crucial to consider given the COVID-19 pandemic has inequitably affected underrepresented, underserved, and socially marginalized individuals (Czeisler et al., 2020; de Bruin, 2020; Novacek et al., 2020; Yancy, 2020). These individuals are forced to cope with the pandemic against a backdrop of systemic inequalities, disenfranchisement from the healthcare system, and increased risk of contracting COVID-19 (Yancy, 2020). There may also be cultural differences in the ways that individuals of different races and ethnicities in the U.S., including Black and Hispanic, cope with COVID19 stress that may influence the association between coping strategies and levels of anxiety, depression and other problems (Brantley et al., 2002; Hill \& Hoggard, 2018; Weiss et al., 2017). Furthermore, recent studies indicate that lower income Americans have experienced greater mental health impacts of COVID-19, suggesting the need to examine income-related differences in stress and coping (McGinty et al., 2020).

\section{Current Study}

To address these gaps in knowledge, we enrolled two community samples of adults to complete an online battery of standardized questionnaires during the early stage of the pandemic. We examined levels of COVID-19 stress, ways of coping with COVID-19 stress, and symptoms of depression and anxiety. We hypothesized that COVID-19-related stress would be positively associated with symptoms of anxiety and depression; and that primary and secondary control coping would be negatively associated with symptoms of anxiety and depression, while disengagement coping would be positively associated with symptoms, beyond the associations of stress with these symptoms. Furthermore, we conducted exploratory analyses to examine whether these associations were moderated by race, ethnicity, and income level.

\section{Methods}

\section{Participants}

Participants included 709 adults ages 18 to 76 years ( $M=38.2$ years, $S D=12.1$ ). Eligible participants were (1) ages 18 years or older and (2) English speaking. As shown in Table 1, the sample was diverse with respect to race $(69 \%$ White, $13 \%$ Asian, 12\% Black, 4\% American Indian), ethnicity (29\% Hispanic), and region of residence (ranging from $15 \%$ from the Northeast to $43 \%$ from the West). The majority of participants reported having a college education (89\%), having children under age $18(61 \%)$, working fulltime (76\%), and earning within the middle-income bracket $(\$ 45,000$ - $\$ 150,000 ; 59 \%)$. Over half (55\%) reported their income was affected by COVID-19 and 13\% reported being unemployed due to COVID-19.

\section{Procedure}

To examine coping and mental health during the early months of the COVID-19 pandemic among a large, diverse sample of U.S. adults, data from two online studies were combined for these analyses. Sample 1 included 572 participants who were recruited from May 5-7, 2020 through Amazon Mechanical Turk (MTurk), an online platform in which people complete tasks in exchange for money. Data were initially collected from 580 people, but five were excluded because they failed attention checks and three were excluded because they completed the measures in less than $10 \mathrm{~min}$, resulting in 572 valid participants. Several studies support the utility of collecting data via MTurk, including for obtaining a diverse sample, obtaining information on mental health problems, and rapidly obtaining data on coping in response to COVID-19 (Buhrmester et al., 2018; Shamblaw et al., 2021). Sample 2 comprised 137 participants who were recruited between April 22 and July 12, 2020 through social media, university emails distribution lists, and ResearchMatch, an online platform created by academic institutions to connect clinical researchers with volunteer participants (Harris et al., 2012; Pulley et al., 2018). After informed consent was obtained, participants completed questionnaires through a secure online data capturing website (Qualtrics in Sample 1 and REDCap in Sample 2). All participants 
Table 1 Participant demographics

\begin{tabular}{|c|c|c|c|c|}
\hline & $\begin{array}{l}\text { Total Sample } \\
(\mathrm{N}=709)\end{array}$ & $\begin{array}{l}\text { Sample 1 } \\
(\mathrm{N}=572)\end{array}$ & $\begin{array}{l}\text { Sample 2 } \\
(\mathrm{N}=137)\end{array}$ & $\mathrm{t} / \chi 2^{a}$ \\
\hline Age in years, $M(S D)$ & $38.2(12.1)$ & $36.9(11.5)$ & $43.4(13.1)$ & $t(1,707)=5.69 * * *$ \\
\hline Sex (\% Female) & 52.5 & 44.9 & 83.2 & $\chi^{2}(1,1)=65.1 * * *$ \\
\hline White/Non-Hispanic (\%) & 56.1 & 50.2 & 81.0 & $\chi 2(1,1)=42.71 * * *$ \\
\hline Race $(\%)$ & & & & $\chi^{2}(7,1)=28.25 * * *$ \\
\hline White/Caucasian & 68.7 & 64.5 & 86.1 & \\
\hline Black/African American & 12.1 & 13.3 & 7.3 & \\
\hline Asian & 13.3 & 15.6 & 3.6 & \\
\hline American Indian/Alaska Native & 4.1 & 4.9 & 0.7 & \\
\hline Mixed race & 1.0 & 0.9 & 1.5 & \\
\hline Other $^{b}$ & 0.8 & 0.7 & 0.9 & \\
\hline Ethnicity (\%) & & & & $\chi^{2}(1,1)=53.5 * * *$ \\
\hline Hispanic or Latino & 28.7 & 34.4 & 5.1 & \\
\hline Not Hispanic or Latino & 71.3 & 65.6 & 94.9 & \\
\hline Education Level (\%) & & & & $\chi 2(3,1)=60.06^{* * *}$ \\
\hline High school diploma or less & 3.0 & 3.1 & 2.2 & \\
\hline Some college/tech & 8.1 & 7.2 & 11.8 & \\
\hline College graduate & 58.8 & 65.4 & 30.9 & \\
\hline Graduate degree & 30.2 & 24.3 & 55.1 & \\
\hline Employment Status (\%) & & & & $\chi 2(2,1)=47.95 * * *$ \\
\hline Working full-time & 75.8 & 80.6 & 55.6 & \\
\hline Working part-time & 11.5 & 10.7 & 14.8 & \\
\hline Not working & 12.7 & 8.8 & 29.6 & \\
\hline Income $(\%)$ & & & & $\chi 2(2,1)=26.46^{* * *}$ \\
\hline$<\$ 45 \mathrm{~K}$ & 34.1 & 35.0 & 29.9 & \\
\hline$\$ 45,000-150,000$ & 59.0 & 60.4 & 52.8 & \\
\hline$\$ 150,000+$ & 6.9 & 4.6 & 17.3 & \\
\hline COVID-19 stress, $M(S D)$ & $37.35(9.2)$ & $37.2(9.5)$ & $38.1(8.2)$ & $t(1,707)=1.02$ \\
\hline Primary control coping, $M(S D)$ & $.18(.06)$ & $.18(.09)$ & $.20(.04)$ & $t(1,698)=3.44 * * *$ \\
\hline Secondary control coping, $M(S D)$ & $.28(.12)$ & $.27(.13)$ & $.28(.06)$ & $t(1,698)=1.44$ \\
\hline Disengagement coping, $M(S D)$ & $.15(.05)$ & $.15(.05)$ & $.14(.03)$ & $t(1,698)==3.34 * * *$ \\
\hline
\end{tabular}

$* * * p<.001$

atest for differences between the two samples

${ }^{\mathrm{b}}$ Includes respondents who chose Other and (in Sample 1 only) Mixed race, Prefer not to answer, and Middle Eastern/North African received compensation for their time and effort. Approval for study procedures was approved by the Institutional Review Board at both participating sites.

\section{Measures}

\section{Demographics}

All participants provided their demographic information including age, sex, race, ethnicity, marital status, education level, employment status, unadjusted gross family income, and financial information related to the recent COVID-19 pandemic.

\section{Stress and Coping}

The Responses to Stress Questionnaire-COVID-19 version (RSQ; Connor-Smith et al., 2000) is a self-report measure that identifies specific sources of stress and coping strategies in response to those stressors. For the current study, participants were provided a list of 14 representative stressors related to COVID-19 (e.g., being diagnosed with COVID19, financial problems because of COVID-19, unable to participate in normal routines) and then asked to rate how stressful each of these stressors was for them in the past 6 months on a 4-point scale from 1 "Not at all" to 4 "Very." They could also indicate "Other" stressor for a total of 15 items. A total stressor score was computed by summing the 
responses to all 15 items, such that higher scores indicated more COVID-19-related stress.

The second portion of the RSQ includes 57 items reflecting three factors of coping and two factors involving automatic responses to COVID-19 related stress. Responses to stress are endorsed on a scale from 1 "Not at all" to 4 "A lot" based on how often the participant used this strategy when they experienced the identified stressors (in this case, COVID-19-related stress). Because of both the controllable and uncontrollable nature of the stressors presented by COVID-19, the present study focused on the three effortful coping factors: primary control coping (i.e., problem-solving, emotional modulation, emotional expression; a sample item is "I do something to try to fix the stressful parts of COVID-19"), secondary control coping (i.e., acceptance, cognitive reappraisal, positive thinking, and distraction, such as "I think about the things I' $m$ learning from COVID-19 or something good that will come from it"), and disengagement coping (i.e., avoidance, denial, and wishful thinking, such as "When I' $m$ around other people I act like COVID-19 never happened"). Using the standard method for scoring the RSQ, proportion scores were calculated for each coping factor by dividing the total score for each of the factors by the total score for the entire RSQ (Connor-Smith et al., 2000). The RSQ has demonstrated excellent internal consistency, test-retest reliability, and convergent and construct validity (Compas et al., 2017). Internal consistency for primary control coping, secondary control coping, and disengagement coping in Sample 1 was $\alpha$ (alpha) $=.84, .83$, and .84 , respectively and $.79, .84$ and .67 , respectively in Sample 2.

\section{Depression Symptoms}

In Sample 1, participants completed the Patient Health Questionnaire (PHQ-9; Kroenke et al., 2010), which is a self-report measure assessing the nine cardinal symptoms of Major Depressive Disorder as established by DSM-IV criteria. Participants were asked to rate each of nine items on a scale from 0 "Not at all" to 3 "Nearly every day" on the basis of how much a symptom bothered them in the past two weeks. Reliability and validity scores from the PHQ-9 have been demonstrated in several large samples (Kroenke et al., 2010). In Sample 2, participants completed the Beck Depression Inventory-II (BDI-II; Beck et al., 1996), which assesses the presence and severity of depressive symptoms during the past two weeks. One item assessing suicidal ideation was removed from the BDI-II because of concerns that the research team could not provide appropriate crisis intervention for those endorsing this item, leaving 20 items that were rated on a 4-point scale from 0 "absence of symptoms" to 3 "severe level of symptoms" (specific wording varies by item). The BDI-II is a well-established and standardized measure that demonstrates good construct validity and internal consistency (Beck et al., 1996). For both depression measures, a total score is calculated by summing the individual items. Internal consistency for the depression measure was $\alpha$ (alpha) $=.92$ for Sample 1 and .93 for Sample 2 . Guided by prior research indicating a strong correlation between these two measures (e.g., Kung et al., 2013) raw sum scores were converted to $z$-scores for each sample separately and then combined to allow for pooled analyses (e.g., Gruhn et al., 2016).

\section{Anxiety Symptoms}

Sample 1 used the 7-item anxiety subscale from the Depression Anxiety Stress Scales (DASS-21; Antony et al., 1998), which assesses autonomic arousal, skeletal muscle effects, situational anxiety, and subjective experience of anxious affect from 0 "did not apply to me at all/Never" to 3 "applied to me very much, or most of the time/Almost Always." The DASS-21 demonstrates good reliability and validity among clinical and non-clinical samples (Davies et al., 2015). Sample 2 used the Beck Anxiety Inventory (BAI; Beck et al., 1988) to assess self-reported subjective, somatic, and panicrelated symptoms of anxiety. Participants are instructed to rate how bothersome each of the 21-items were for them during the past month on a scale from 0 "Not at all" to 3 "Severely, it bothered me a lot." The BAI demonstrates good reliability and validity (Beck et al., 1988). For both anxiety measures, a total score is calculated by summing the individual items. Internal consistency for the anxiety measure was $\alpha$ (alpha) $=.93$ for Sample 1 and .93 for Sample 2. Guided by prior research indicating a strong correlation between these two measures (e.g., Norton, 2007), raw sum scores were converted to $z$-scores for each sample separately and then combined to allow for pooled analyses (Gruhn et al., 2016).

\section{Statistical Analyses}

All analyses were conducted using IBM SPSS version 27. Bivariate correlations among age, coping factors, levels of stress, and symptoms of depression and anxiety are presented in Table 2 . Independent Samples $t$-tests were computed to investigate sex, racial, ethnic and income differences in levels of stress, coping strategies, and symptoms of depression and anxiety. Multiple linear regression was used to examine stress and coping factors as predictors of depression and anxiety, controlling for age, sex, race (Black v. non-Black), ethnicity (Hispanic v. non-Hispanic), low income ( $\$ 45 \mathrm{~K}$ or less in annual income $\mathrm{v}$. greater than $\$ 45 \mathrm{~K}$ annual income), and sample (1 or 2). The PROCESS macro v3.5 (Hayes \& Rockwood, 2017) for SPSS was then used to explore whether these associations were moderated by race, ethnicity and income (Table 3). PROCESS provides mean centering of products and bootstrapped confidence intervals for 
Table 2 Descriptive statistics and correlations among age, stress, coping and symptoms of anxiety and depression

\begin{tabular}{lllllllll}
\hline Variable & Mean (SD) & 1 & 2 & 3 & 4 & 5. & 6 & 7 \\
\hline 1. Age & $38.18(12.10)$ & - & & & & & & \\
2. COVID-19 Stress & $37.35(9.22)$ & $-.15^{* *}$ & - & & & & \\
3. Primary Control Coping & $0.18(0.06)$ & $.27^{* *}$ & $-.29^{* *}$ & - & & & \\
4. Secondary Control Coping & $0.28(0.12)$ & $.24^{* *}$ & $-.56^{* *}$ & $.42^{* *}$ & - & & & \\
5. Disengagement Coping & $0.15(0.05)$ & $-.14^{* *}$ & $.24^{* *}$ & $-.58^{* *}$ & $-.51^{* *}$ & - & & \\
6. Depression z-Score & $0.0(1.0)$ & $-.22^{* *}$ & $.64^{* *}$ & $-.50^{* *}$ & $-.60^{* *}$ & $.34^{* *}$ & - & \\
7. Anxiety $z$-score & $0.0(1.0)$ & $-.23^{* *}$ & $.66^{* *}$ & $-.44^{* *}$ & $-.61^{* *}$ & $.29^{* *}$ & $.77^{* *}$ & - \\
\hline
\end{tabular}

$* * p<.01$

interaction terms. Significant interactions were probed with simple slopes analyses to determine the nature of any moderating relationships.

\section{Results}

\section{Preliminary Analyses}

Before combining data from the two samples, participants from Samples 1 and 2 were compared on age, sex, race, ethnicity, education, income, stress and coping. As shown in Table 1, participants from Sample 2 were significantly older, more likely to be female, white-non-Hispanic, and to have completed college, and less likely to be working full-time; they reported greater use of primary control coping and less use of disengagement coping. Therefore, a dichotomous variable indicating sample was included as a control in all multiple variable analyses.

On the COVID-19-related stressors list, participants reported a mean item score of 2.46 , corresponding to "somewhat stressful", indicating that on average participants were at least somewhat bothered by multiple stressors. Applying PHQ-9 cutoffs provided by Kroenke et al. (2010), the proportion of participants in Sample 1 whose depression scores fell into established clinical cutoffs was $24.3 \%$ moderate, $27.9 \%$ moderately severe, and $11.9 \%$ severe. Applying BDI-II cutoffs provided by Beck et al. (1996), the proportion of participants in Sample 2 whose depression scores fell into established clinical cutoffs was $10.9 \%$ in the moderate range and $16.5 \%$ in the severe range. Regarding anxiety, in Sample 1, the proportion of participants whose DASS21 anxiety scores fell into established clinical cutoffs (Antony et al., 1998) was $35.2 \%$ in the moderate range and $21.3 \%$ in the severe range. In Sample 2, the proportion of participants whose BAI anxiety scores fell into established clinical cutoffs (Beck et al., 1988) was $15.3 \%$ in the moderate range and $5.5 \%$ in the severe range.

\section{Bivariate Correlations and Comparisons}

Table 2 shows correlations among key study variables. As expected, at the bivariate level primary and secondary control coping were negatively related to COVID-19-related stress, anxiety, and depression ( $r$ 's range from -.29 to -.61 , all $p<.01$ ), whereas disengagement coping was positively related to these variables ( $r$ 's range from .24 to .34 , all $p<.01$ ). Furthermore, COVID-19-related stress, depression, and anxiety were positively correlated with one another ( $r$ 's range from .64 to .77 , all $p<.01$ ). Regarding demographic variables, age was positively correlated with primary control coping and secondary control coping, and negatively correlated with disengagement coping, stress, depression and anxiety (all $p<.05$, ranging from small to medium in magnitude). Female participants reported more use of primary and secondary control coping, less use of disengagement coping, less COVID19-related stress, and less depression and anxiety than male participants (all $p<.01$; see Supplemental table). Participants who identify as Black, and (separately) who identify as Hispanic, reported less use of secondary control coping, more use of disengagement coping, more COVID-19-related stress, and more depression and anxiety than those who identify as non-Black and non-Hispanic, respectively (all $p<.05$ ). Furthermore, Hispanic participants reported less use of primary control coping than non-Hispanic participants. Although participants who fell into the low-income bracket versus the middle/high income bracket did not differ significantly in COVID19 stress, those in the low-income bracket experienced more depression and anxiety and used less primary control coping compared to individuals with middle/high income. Based on these differences, multivariable analyses included effects for age, sex, race and ethnicity, income, and sample source.

\section{Multiple Regression Analyses: COVID-19 Stress and Coping as Predictors or Symptoms of Anxiety and Depression}

Stepwise linear multiple regression analyses were conducted to examine associations between levels of COVID-19 related stress, coping, and symptoms of anxiety and depression. In the first step (Table 3, Model 1), COVID-19 stress was entered as a predictor of symptoms, controlling for age, sex, race, ethnicity, income and sample. The models for anxiety symptoms $\left(F[7676]=95.15, p<.001\right.$, adjusted $\left.R^{2}=.49\right)$ 
Table 3 Main effects and moderators of coping strategies as predictors of anxiety and depression symptoms

\begin{tabular}{|c|c|c|c|c|c|c|c|c|c|c|}
\hline & \multicolumn{5}{|c|}{ Anxiety } & \multicolumn{5}{|c|}{ Depression } \\
\hline & $B$ & $95 \% C I$ & $t$ & $p$ & $\Delta R_{a d j}^{2}$ & $B$ & $95 \% C I$ & $t$ & $p$ & $\Delta R_{a d j}^{2}$ \\
\hline 1.COVID-19 Stress & .06 & $.056, .069$ & 19.96 & $<.001$ & $R^{2}=.49$ & .06 & $.056, .068$ & 18.92 & $<.001$ & $R^{2}=.45$ \\
\hline 2.COVID-19 Stress & .06 & $.052, .064$ & 18.70 & $<.001$ & - & .06 & $.049, .061$ & 17.71 & $<.001$ & - \\
\hline Primary Control Coping (PCC $)^{\mathrm{a}}$ & -3.38 & $-4.27,-2.49$ & -7.45 & $<.001$ & $.03 * * *$ & -4.81 & $-5.71,-3.91$ & -10.54 & $<.001$ & $.07 * * *$ \\
\hline \multicolumn{11}{|l|}{ 3.PCC x Moderator: } \\
\hline Race & -1.84 & $-4.82,1.15$ & -1.21 & .23 & .001 & -1.27 & $-4.28,1.74$ & -.83 & .41 & .0005 \\
\hline Ethnicity & 3.17 & $.23,6.10$ & 2.12 & .03 & $.003 *$ & 1.00 & $-1.96,3.97$ & .66 & .51 & .0003 \\
\hline Low income & 1.35 & $-.36,3.06$ & 1.55 & .12 & .002 & .02 & $-1.71,1.75$ & .02 & .98 & $<.0001$ \\
\hline 2.COVID-19 Stress & .05 & $.038, .052$ & 13.25 & $<.001$ & - & .04 & $.037, .051$ & 12.39 & $<.001$ & - \\
\hline Secondary Control Coping (SCC) ${ }^{a}$ & -2.67 & $-3.20,-2.14$ & -9.96 & $<.001$ & $.07 * * *$ & -2.175 & $-3.27,-2.16$ & -9.63 & $<.001$ & $.06 * * *$ \\
\hline \multicolumn{11}{|l|}{ 3.SCC x Moderator: } \\
\hline Race & -3.00 & $-5.11,-.89$ & -2.79 & .006 & $.005 * *$ & -2.87 & $-5.09,-.66$ & -2.54 & .01 & $.005 *$ \\
\hline Ethnicity & .12 & $-1.13,1.37$ & .19 & .85 & $<.0001$ & -.26 & $-1.57,1.06$ & -.38 & .70 & .0001 \\
\hline Low income & .08 & $-.82, .99$ & .18 & .86 & $<.0001$ & -.13 & $-.1 .09, .82$ & -.28 & .78 & .0001 \\
\hline 2.COVID-19 Stress & .06 & $.054, .066$ & 19.03 & $<.001$ & - & .06 & $.05, .06$ & 17.88 & $<.001$ & - \\
\hline Disengagement Coping (DC) ${ }^{\mathrm{a}}$ & 2.30 & $1.19,3.41$ & 4.07 & $<.001$ & $.01 * * *$ & 3.715 & $2.57,4.86$ & 6.39 & $<.001$ & $.03 * * *$ \\
\hline \multicolumn{11}{|l|}{ 3.DC x Moderator: } \\
\hline Race & -2.85 & $-6.28, .55$ & -1.65 & .10 & .002 & -.36 & $-6.87, .15$ & -1.88 & .06 & .003 \\
\hline Ethnicity & -5.12 & $-8.07,-2.17$ & -3.41 & $<.001$ & $.008 * * *$ & -2.19 & $-5.25, .87$ & -1.40 & .16 & .002 \\
\hline Low income & -.84 & $-3.04,1.35$ & -.75 & .45 & .0004 & .94 & $-1.31,3.20$ & .82 & .41 & .0005 \\
\hline
\end{tabular}

All analyses control for age, sex, race, ethnicity, low income, and sample source. $\mathrm{B}=$ unstandardized regression coefficients. $\Delta \mathrm{R}^{2}$ for Model 2 indicates the total variance in depression or anxiety explained by coping + control variables, above and beyond the adjusted $R^{2}$ for model 1 . $R^{2}$ for moderators indicates additional variance explained by the moderator, above and beyond the adjusted $\mathrm{R}^{2}$ for model 2 . For moderation, reference categories were as follows: race (Black [ref.] versus non-Black), Ethnicity (Hispanic [ref.] versus non-Hispanic), Low income $(<45 \mathrm{~K}$ annual income [ref.] versus more)

Model 1 includes control variables and stress; Model 2 adds coping; Model 3 adds interaction terms for coping x demographic variable

*R-squared change significant at $p<.05$ compared to prior Model

**R-squared change significant at $p<.01$ compared to prior Model

***R-squared change significant at $p<.001$ compared to prior Model

When we applied the False Discovery Rate (FDR; Benjamini \& Hochberg, 2000) correction for multiple analyses, the pattern of results was generally unchanged, such that stress and all three forms of coping remained significant predictors of both anxiety and depression, and three out of four interactions terms remained significant. Only the interaction of ethnicity x primary control coping in relation to anxiety was no longer significant. Similar results were obtained using the more conservative Bonferroni correction for multiple analyses (only the interactions of ethnicity $\mathrm{x}$ primary control coping in relation to anxiety, and race $\mathrm{x}$ secondary control coping in relation to depression, were no longer significant)

and depression symptoms $(F[7675]=79.93, p<.001$, adjusted $R^{2}=.45$ ) were significant. As expected, adults who reported more COVID-19-related stress reported more anxiety and depression symptoms. ${ }^{1}$

In the next step we added coping strategies to the above model as predictors of symptoms, after controlling for stress and demographic variables. Separate models were estimated for primary control coping, secondary control coping, and disengagement coping. The overall equations predicting anxiety symptoms were significant for primary control $(F(8,675)=96.91 .87, p<.001)$, secondary control

\footnotetext{
1 These multivariable associations between stress and symptoms were significant for both samples separately (analyses not shown) as well as for the combined sample reported here.
}

$(F(8,675)=107.73, p<.001)$, and disengagement coping, $(F(8,675)=87.25, p<.001)$. Similarly, the models predicting depression symptoms were significant for primary control $(F(8,674)=95.06, p<.001)$, secondary control $(F$ $(8,674)=91.03, p<.001)$, and disengagement coping $(F$ $(8,674)=79.17, p<.001)$. Greater use of primary control and secondary control coping was associated with lower levels of depression and anxiety, whereas greater use of disengagement coping was associated with higher symptom levels of both depression and anxiety. ${ }^{2}$ As shown in in the $\Delta R^{2}$ column of Table 3 (Model 2), in all cases coping strategies

\footnotetext{
2 These multivariable associations between coping and symptoms were significant for both samples separately (analyses not shown) as well as for the combined sample reported here.
} 
a.

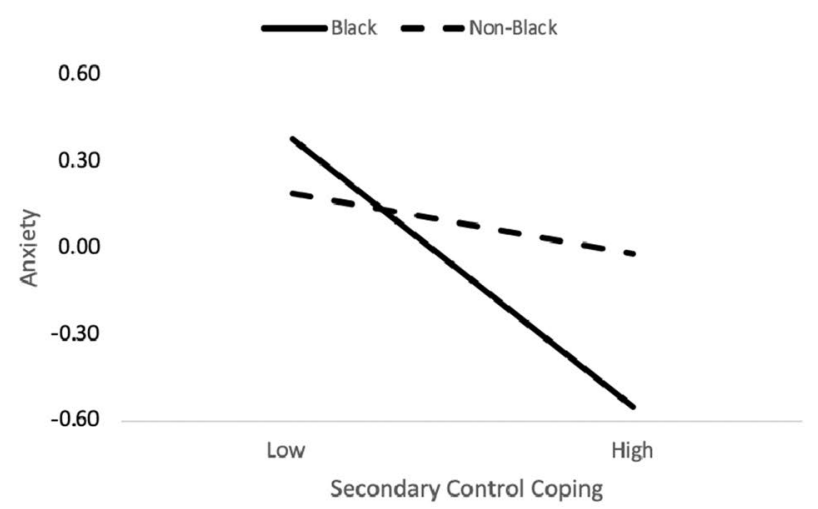

b.

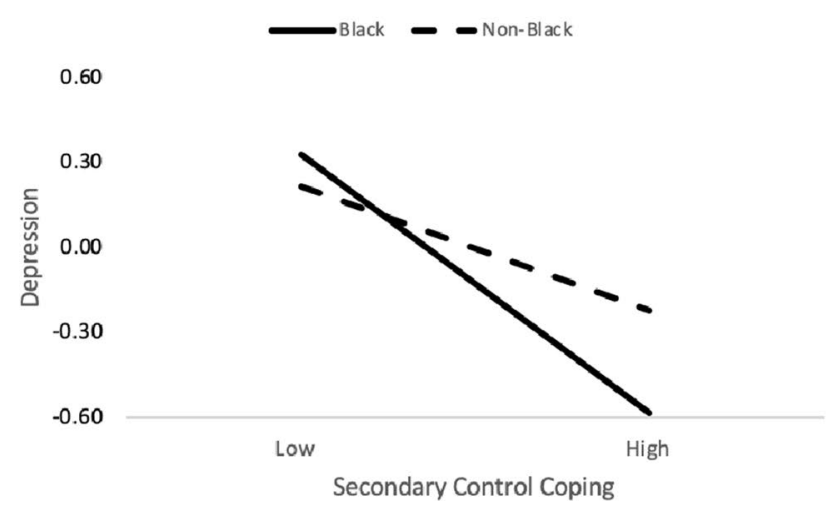

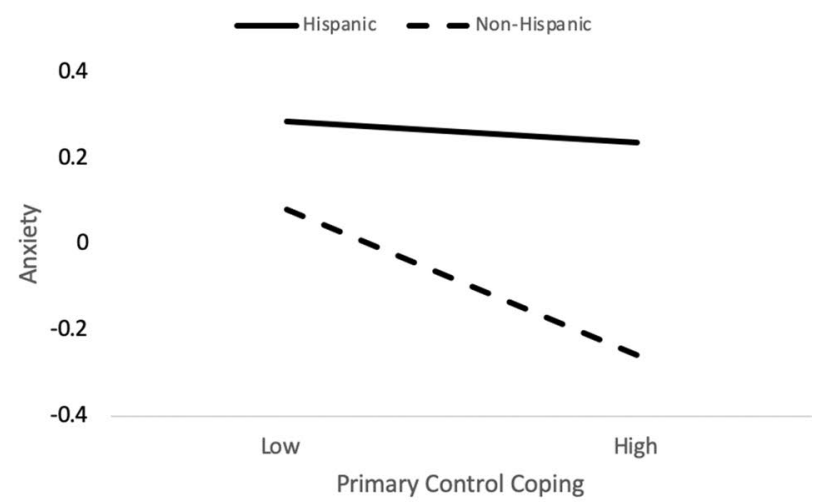

d.

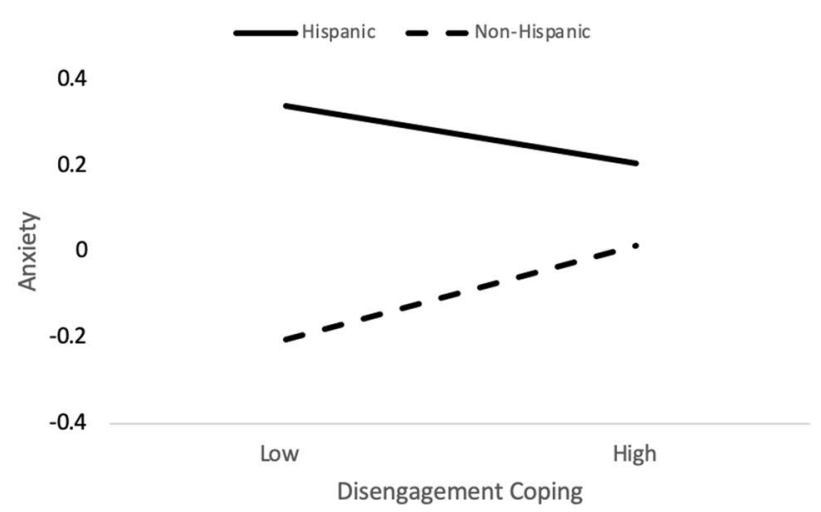

Fig. 1 Race and ethnicity as moderators of the association between coping and symptoms

explained additional variability in symptoms, beyond that explained by stress, although effect sizes were small.

Next, we examined moderators of these associations between coping and symptoms using the PROCESS macro for SPSS (Hayes \& Rockwood, 2017). As shown in Table 3, Model 3, race emerged as a significant moderator, such the association between secondary control coping and both anxiety and depression was significantly stronger for participants who identified as Black versus all other races (see Fig. 1a for anxiety and Fig. 1b for depression). Second, ethnicity moderated the association between coping and anxiety, such that the association of both primary control coping (Fig. 1c) and disengagement coping (Fig. 1d) with anxiety was significant for non-Hispanic but nonsignificant for Hispanic participants.

\section{Discussion}

The COVID-19 global pandemic has presented an international public health crisis that has brought unprecedented levels of financial, social, and health-related stress, as well as high rates of mental health problems, including symptoms of anxiety, depression, trauma, and substance abuse (e.g., Czeisler et al., 2020; McKay \& Asmundson, 2020). Individuals of color and otherwise marginalized populations are at particularly high risk for adverse outcomes (de Bruin, 2020; Novacek et al., 2020). The need for resources to address these mental health problems is a public health priority (Gruber et al., 2020; Kaslow et al., 2020); however, current evidence on ways to cope with COVID-19-related stress has not been informed by theories that describe a full range of coping strategies that may be effective. The current study addressed this gap by examining associations among COVID-19related stress, three empirically-validated types of coping strategies, and symptoms of anxiety and depression, in a large sample of U.S. adults in the early stages of the pandemic. These analyses are guided by a controlbased model of coping that has identified adaptive and maladaptive ways of coping with a range of controllable and uncontrollable stressors (Compas et al., 2017; Weisz et al., 1994). 
Consistent with recent findings (e.g., Kujawa et al., 2020; Taylor et al., 2020), these adults reported significant levels of stress related to multiple facets of the COVID-19 pandemic. These include financial difficulties, difficulty balancing work and family responsibilities, social isolation, and fears about themselves or family members becoming sick with COVID-19. Participants also reported elevated symptoms of both anxiety and depression; specifically, more than a quarter of the sample reported moderate to severe levels of depression symptoms, and more than one-fifth reported moderate to severe symptoms of anxiety. These high symptom levels are consistent with those reported in two other recent studies of U.S. adults during the pandemic (Czeisler et al., 2020; McGinty et al., 2020). In keeping with earlier reports (e.g., Krogstad \& Lopez, 2020; Kujawa et al., 2020; Millett et al., 2020), the current analyses identified noteworthy subgroup variations in stress and symptom levels: frequency and severity of stressors was higher among adults who identified as Black or Hispanic, and symptoms were higher among those who identified as Black, Hispanic, or low-income. These differential patterns of stress exposure suggest that public health interventions designed to reduce COVID-19-related stress should be targeted to these vulnerable populations (Kaslow et al., 2020).

Unexpectedly, males and younger adults reported more COVID-19-related stress, and higher symptom levels. Despite older adults being at higher risk for longer and more severe course of the illness and typically being more isolated during the pandemic, our findings coincide with recent data indicating that younger adults report more anxiety and social isolation than older adults during this period (de Bruin, 2020; McGinty et al., 2020). Socioemotional selectivity theory suggests the size of social networks typically declines with older age, while satisfaction with these networks increases (English \& Carstensen, 2014); thus, older adults who are required to isolate to reduce exposure to COVID-19 may experience this isolation less negatively. The finding that males reported more stress and symptoms is surprising, as the opposite pattern has typically been observed both prior to (Kessler et al., 2005) and during the pandemic (Kujawa et al., 2020). This unexpected finding may reflect that in our diverse sample, males were younger and more likely to be Black or Hispanic than females, or that more distressed individuals chose to complete the online surveys.

Multivariable models indicated that COVID-19-related stress is significantly and positively associated with higher levels of anxiety and depression. These associations were large in magnitude and underscore that variations in the ways that adults experience the pandemic (e.g., extent to which it affects their income, family life and social functioning) are related in meaningful ways to their symptom levels.
In the primary focus of this study, we examined associations of three types of coping strategies with symptoms of anxiety and depression, above and beyond the association of stress with symptoms. Consistent with a large body of research supporting the control-based model of coping, efforts to cope with COVID-related stress by engaging with the stressor (whether through primary or secondary control coping) were associated with lower symptom levels. These patterns have recently been confirmed with other samples of adults coping with COVID-19 (e.g., Gurvich et al., 2020; Shamblaw et al., 2021). Although effect sizes were small, the current findings suggest that stress associated with COVID-19 is heterogenous in nature, including aspects that are perceived as controllable and uncontrollable. Furthermore, disengagement coping, which involves efforts to avoid or deny the stressor, was strongly associated with higher symptom levels for the entire sample. The consistency of these associations between coping and symptoms, even after controlling for stress levels, suggests that all three forms of coping may be important in understanding how to build resilience in response to COVID-19 stress. Teaching the use of a range of coping skills, including problem solving, the modulated expression of emotions, cognitive reappraisal, and acceptance, as well as skills to appraise accurately the controllability of specific stressors (e.g., Bettis et al., 2017; Compas et al., 2010) all may be important in the management of COVID-19 stress. Conversely, efforts to deny or avoid COVID-19-related stress may be maladaptive (Aldao et al., 2010), in part because of the pervasive spread of the virus, restrictions due to stay-at-home guidelines, and loss of employment, which make the stress of COVID-19 unavoidable. Individuals facing chronic stress may simultaneously use both adaptative and maladaptive coping skills depending on both the environmental context (dangerous or normative) and the outcome (safety or injury; Wadsworth, 2015). Additional research is needed on potential interactions among these forms of coping; for example, when individuals employ multiple coping strategies simultaneously, might disengagement coping moderate or negate the benefits of primary or secondary control forms of coping? Does perceived controllability of the stressor moderate associations between coping and symptoms?

The current sample provided sufficient power to examine whether associations between coping and symptoms were moderated by theoretically important demographic variables. Two interesting patterns emerged. First, efforts to adapt to COVID-19-related stress by using forms of secondary control coping, including reframing one's thoughts or accepting the stressor, had a stronger association with lower symptoms of depression and anxiety for Black than non-Black adults. In other words, coping strategies that are best suited to uncontrollable stressors were particularly 
beneficial for the mental health of participants who identify as Black. Throughout the pandemic, people of color have experienced higher rates, severity and mortality of COVID-19 (Centers for Disease Control and Prevention, 2021), accompanied with limited access to testing and vaccines (Grigsby-Toussaint et al., 2021) and to culturally sensitive sources of support (DeSouza et al.. 20,201). These health disparities, against a backdrop of persistent systemic racism, create a public health crisis for Black individuals (American Public Health Association, 2020) that is likely perceived as uncontrollable. Second, although Hispanic participants reported less use of primary control coping and more use of disengagement coping than non-Hispanic participants, these coping strategies were not associated with symptoms of anxiety for this group. In the context of high and persistent cumulative stress levels, typically-maladaptive coping pattens such as disengagement may not add to the strong negative effects of stressors on mental health, and may actually be functionally adaptive in the short term (Amirkhan, 2020; Wadsworth, 2015). These race/ethnicity by coping interactions suggest that intervention designed to increase adaptive coping and reduce the negative mental health consequences of COVID-19 should be culturally adapted (Rathod et al., 2018).

This study has several limitations including a reliance on self-report measures; a cross-sectional design with data collected early in the pandemic such that we could not observe changes over time in stress, coping patterns or symptoms; use of on-line platforms to collect data rapidly (Cheung et al., 2017); and that the two samples combined here used different measures of depression and anxiety symptoms. Additional research is needed to examine responses to the COVID-19 pandemic over time, including whether perceptions of controllability have changed as vaccines and therapeutic medications have become more widely available, and to establish causal links between coping and symptoms. Researchers should also examine how other psychosocial aspects of the pandemic such as risk perception (Gerhold, 2020) may interact with coping to influence outcomes. Despite these limitations, these findings from a large heterogeneous sample of $U$. S. adults have a number of implications for public health and clinical interventions. First, a noteworthy portion of the sample reported significant levels of depression and anxiety symptoms that indicate the need for mental health interventions, consistent with prior research (e.g., Czeisler et al., 2020). Second, the findings underscore that stress and emotional distress related to COVID-19 are not uniformly distributed across the population; rather, certain subgroups are more vulnerable to adverse outcomes, including people of color, whose greater risk for health and economic consequences are well documented, and younger adults, who are sometimes portrayed in the media as relatively unaffected. Identification of subgroups at greater risk is a first step toward targeted preventive interventions (Kaslow et al., 2020). Finally, the finding that both primary and secondary control coping are associated with reduced risk for symptoms suggests that evidence-based treatments that teach emotion regulation (e.g., Dialectical Behavior therapy; Linehan, 1993), acceptance (e.g., Acceptance and Commitment Therapy; Hayes et al., 1999), and cognitive restructuring (e.g., Cognitive Behavior Therapy; Beck et al., 1979) may also be effective in reducing the impact of COVID-19-related stress and thus reducing anxiety and depression. Increasing the accessibility of interventions through novel modes of service delivery such as telehealth or integrated primary care models is imperative in order to reduce the devastating public mental health consequences of this and future pandemics (Pfefferbaum \& North, 2020).

Supplementary Information The online version contains supplementary material available at https://doi.org/10.1007/s12144-021-02444-6.

Acknowledgements We would like to acknowledge the research assistance of Jennifer Meigs, B.A., as well as support from a gift from Patricia and Rodes Hart, grant T32-MH18921 from the National Institute of Mental Health, and grants UL1TR000445, U24TR00157 9, and 5 U24 TR001579-02 from the National Institutes of Health (NIH) Clinical and Translational Science Award (CTSA) program.

Authors' Contributions All authors made substantial contributions to the conception or design of the work; the acquisition, analysis, or interpretation of data; and/or the writing and revision of the manuscript. All authors approved the final version to be published.

Funding Partial financial support was provided by a gift from Patricia and Rodes Hart, grant T32-MH18921 from the National Institute of Mental Health and grants UL1TR000445, U24TR00157 9, and 5 U24 TR001579-02 from the National Institutes of Health (NIH) Clinical and Translational Science Award (CTSA) program.

Data Availability The datasets generated during and analyzed during the current study are not publicly available but are available from the corresponding author on reasonable request.

\section{Code Availability N/A}

\section{Declarations}

\section{Conflict of Interest None.}

Ethics Approval Approval for study procedures was approved by the Institutional Review Board at Loyola University Maryland (HS-2020053) and Vanderbilt University (IRB-200726).

Consent to Participate All participants provided informed consent.

Consent for Publication All participants agreed that their deidentified data could be used for publication. 


\section{References}

Ahuja, K. K. (2021). Scanning the VIRUS: A study of dimensions of stress and coping with COVID-19. Current Psychology. https:// doi.org/10.1007/s12144-021-01369-4

Aldao, A., Nolen-Hoeksema, S., \& Schweizer, S. (2010). Emotionregulation strategies across psychopathology: A meta-analytic review. Clinical Psychology Review, 30, 217-237. https://doi. org/10.1016/j.cpr.2009.11.004

Amirkhan, J. H. (2020). Stress overload in the spread of coronavirus. Anxiety, Stress, and Coping. https://doi.org/10.1080/10615806. 2020.1824271

Antony, M. M., Bieling, P. J., Cox, B. J., Enns, M. W., \& Swinson, R. P. (1998). Psychometric properties of the 42-item and 21-item versions of the depression anxiety stress scales in clinical groups and a community sample. Psychological Assessment, 10(2), 176-181. https://doi.org/10.1037/1040-3590.10.2.176

Asmundson, G. J. G., Paluszek, M. M., Landry, C. A., Rachor, G. S., McKay, D., \& Taylor, S. (2020). Do pre-existing anxietyrelated and mood disorders differentially impact COVID-19 stress responses and coping? Journal of Anxiety Disorders, 74, 102271. https://doi.org/10.1016/j.janxdis.2020.102271

Beam, C. R., \& Kim, A. J. (2020). Psychological sequelae of social isolation and loneliness might be a larger problem in young adults than older adults. Psychological Trauma Theory Research Practice and Policy, 12(S1), S58-S60. https://doi.org/10.1037/tra0000774

Bearman, S. K., \& Weisz, J. R. (2009). Primary and secondary control enhancement training: Applying the deployment-focused model of treatment development and testing. In C. A. Essau (Ed.), Treatments for adolescent depression: Theory and practice (pp. 97-122). Oxford University Press.

Beck, A. T., Rush, A. J., Shaw, B. F., \& Emery, G. (1979). Cognitive therapy of depression. Guilford Press.

Beck, A. T., Epstein, N., Brown, G., \& Steer, R. A. (1988). An inventory for measuring clinical anxiety: Psychometric properties. Journal of Consulting and Clinical Psychology, 56(6), 893-897. https://doi.org/10.1037/0022-006X.56.6.893

Beck, A. T., Steer, R. A., \& Brown, G. K. (1996). Manual for the Beck depression inventory-II. Psychological Corporation.

Benjamini, Y., \& Hochberg, Y. (2000). On the adaptive control of the false discovery rate in multiple testing with independent statistics. Journal of Educational and Behavioral Statistics, 25, 60-83. https://doi.org/10.3102/10769986025001060

Bettis, A., Coiro, M. J., *England, J., Murphy, L. K., Zelkowitz, R. L., Dejardins, L ... Compas, B. E. (2017). Comparison of two approaches to prevention of mental health problems in college students: Enhancing coping and executive function skills. Journal of American College Health, 65, 313-322. https://doi.org/ 10.1080/07448481.2017.1312411.

Brantley, P. J., O’Hea, E. L., Jones, G., \& Mehan, D. J. (2002). The influence of income level and ethnicity on coping strategies. Journal of Psychopathology and Behavioral Assessment, 24, 39-45. https://doi.org/10.1023/A:1014001208005

Centers for Disease Control and Prevention. (2021). Risk for COVID-19 infection, hospitalization, and death by race/ethnicity. https://www. cdc.gov/coronavirus/2019-ncov/covid-data/investigations-disco very/hospitalization-death-by-race-ethnicity.html

Cheung, J. H., Burns, D. K., Sinclair, R. R., \& Sliter, M. (2017). Amazon mechanical Turk in organizational psychology: An evaluation and practical recommendations. Journal of Business and Psychology, 32(4), 347-361. https://doi.org/10.1007/s10869-016-9458-5

Compas, B. E., Stoll, M. F., Thomsen, A. H., Oppedisano, G., Epping-Jordan, J. E., \& Krag, D. N. (1999a). Adjustment to breast cancer: Age-related differences in coping and emotional distress. Breast Cancer Research and Treatment, 54, 195-203.
Compas, B. E., Stoll, M. F., Thomsen, A. H., Oppedisano, G., Epping-Jordan, J. E., \& Krag, D. N. (1999b). Adjustment to breast cancer: Age-related differences in coping and emotional distress. Breast Cancer Research and Treatment, 54, 195-203.

Compas, B. E., Champion, J. E., Forehand, R., Cole, D. A., Reeslund, K. L., Fear, J., Hardcastle, J., Keller, G., Rakow, A., Garai, E., Merchant, M. J., \& Roberts, L. (2010). Coping and parenting: Mediators of 12-month outcomes of a family group cognitive-behavioral preventive intervention with families of depressed parents. Journal of Consulting and Clinical Psychology, 78(5), 623-634.

Compas, B. E., Bemis, H., Gerhardt, C. A., Dunn, M. J., Rodriguez, E. M., Desjardins, L., Rodriguez, E., \& Vannatta, K. (2015). Mothers and fathers coping with their children's cancer: Individual and interpersonal processes. Health Psychology, 34(8), 783-793. https://doi.org/10.1037/hea0000202

Compas, B. E., Jaser, S. S., Bettis, A. H., Watson, K. H., Gruhn, M., Dunbar, J. P., Williams, E., \& Thigpen, J. C. (2017). Coping, emotion regulation, and psychopathology in childhood and adolescence: A meta-analytic and narrative review. Psychological Bulletin, 143, 939-991. https://doi.org/10.1037/ bu10000110

Connor-Smith, J. K., Compas, B. E., Wadsworth, M. E., Thomsen, A. H., \& Saltzman, H. (2000). Responses to stress in adolescence: Measurement of coping and involuntary responses to stress. Journal of Consulting and Clinical Psychology, 68, 976-992. https://doi.org/10.1037/0022-006X.68.6.976

Czeisler, M. E., Lane, R. I., Petrosky, E., Wiley, J. F., Christensen, A., Njai, R., Weaver, M. D., Robbins, R., Facer-Childs, E. R., Barger, L. K., Czeisler, C. A., Howard, M. E., \& Rajaratnam, S. M. W. (2020). Mental health, substance use, and suicidal ideation during the COVID-19 pandemic - United States, June 24-30, 2020. MMWR. Morbidity and Mortality Weekly Report, 69, 1049-1057. https://doi.org/10.15585/mmwr.mm6932a1

Davies, G., Caputi, P., Skarvelis, M., \& Ronan, N. (2015). The depression anxiety and stress scales: References data from a large psychiatric outpatient sample. Australian Journal of Psychology, 67(2), 97-104. https://doi.org/10.1111/ajpy.12069

de Bruin, W. B. (2020). Age differences in COVID-19 risk perceptions and mental health: Evidence from a national U.S. survey conducted in march 2020. The Journals of Gerontology: Series B. https://doi.org/10.1093/geronb/gbaa074

English, T., \& Carstensen, L. L. (2014). Emotional experience in the mornings and the evenings: Consideration of age differences in specific emotions by time of day. Frontiers in Psychology, 5, Article 185. https://doi.org/10.3389/fpsyg.2014. 00185

Finkelstein-Fox, L., \& Park, C. L. (2019). Control-coping goodnessof-fit and chronic illness: A systematic review of the literature. Health Psychology Review, 13(2), 137-162. https://doi.org/10. 1080/17437199.2018.1560229

Gao, J., Zheng, P., Jia, Y., Chen, H., Mao, Y., Chen, S., Wang, Y., Fu, H., \& Dai, J. (2020). Mental health problems and social media exposure during COVID-19 outbreak. PLoS One, 15(4), 1-10. https://doi.org/10.1371/journal.pone.0231924

Gerhold, L. (2020). COVID-19: Risk perception and coping strategies. PsyArXiv. https://doi.org/10.31234/osf.io/xmpk4

Gruber, J., Prinstein, M., Abramowitz, J. S., Albano, A. M., Aldao, A., Borelli, J., Chung, T., Clark, L. A., Davila, J., Forbes, E. E., Gee, D. G., Nagayama Hall, G. C., Hallion, L. S., Hinshaw, S. P., Hofmann, S. G., Holon, S. D., Joormann, J., Kazdin, A. E., Klein, D. N., ... Weinstock, L. (2020). Mental health and clinical psychological science in the time of COVID-19: Challenges, opportunities, and a call to action. American Psychologist. https://doi.org/10.1037/amp0000707. 
Gruhn, M. A., Dunbar, J. P., Watson, K. H., Reising, M. M., McKee, L., Forehand, R., Cole, D. A., \& Compas, B. E. (2016). Testing specificity among parents' depressive symptoms, parenting, and child internalizing and externalizing symptoms. Journal of Family Psychology, 30, 309-319.

Gurvich, C., Thomas, N., Thomas, E. H., Hudaib, A.-R., Sood, L., Fabiatos, K., Sutton, K., Isaacs, A., Arunogiri, S., Sharp, G., \& Kulkarni, J. (2020). Coping styles and mental health in response to societal changes during the COVID-19 pandemic. International Journal of Social Psychiatry. https://doi.org/10.1177/0020764020 961790

Harris, P. A., Scott, K. W., Lebo, L., Hassan, N., Lightner, C., \& Pulley, J. (2012). ResearchMatch: A national registry to recruit volunteers for clinical research. Academic Medicine, 87, 66-73. https://doi. org/10.1097/ACM.0b013e31823ab7d2

Hayes, A. F., \& Rockwood, N. J. (2017). Regression-based statistical mediation, moderation, and conditional process analysis: Observations, recommendations, and implementation. Behaviour Research and Therapy, 98, 39-57. https://doi.org/10.1016/j.brat.2016.11. 001

Hayes, S. C., Strosahl, K., \& Wilson, K. G. (1999). Acceptance and commitment therapy: An experiential approach to behavior change. Guilford Press.

Kaslow, N. J., Friis-Healy, E. A., Cattie, J. E., Cook, S. C., Crowell, A. L., Cullum, K. A., del Rio, C., Marshall-Lee, E. D., Lo Pilato, A. M., VanderBroek-Stice, L., Ward, M. C., White, D. T., \& Farber, E. W. (2020). Flattening the emotional distress curve: A behavioral health pandemic response strategy for COVID-19. American Psychologist. Advance online publication. https://doi.org/10.1037/ amp0000694.

Kessler, R. C., Chiu, W. T., Demler, A., \& Walters, E. E. (2005). Prevalence, severity, and comorbidity of twelve-month DSM-IV disorders in the National Comorbidity Survey Replication (NCS-R). Archives of General Psychiatry, 62, 617-627. https://doi.org/10. 1001/archpsyc.62.6.617

Kroenke, K., Spitzer, R. L., Williams, J. B., \& Löwe, B. (2010). The patient health questionnaire somatic, anxiety, and depressive symptom scales: A systematic review. General Hospital Psychiatry, 32(4), 345-359. https://doi.org/10.1016/j.genhosppsych. 2010.03.006

Krogstad, J. M., \& Lopez, M. H. (2020, August 04). Coronavirus economic downturn has hit Latinos especially hard. Pew Research Center. https://www.pewresearch.org/hispanic/2020/08/04/coron avirus-economic-downturn-has-hit-latinos-especially-hard/

Kujawa, A., Green, H., Compas, B., Dickey, L., \& Pegg, S. (2020). Exposure to COVID-19 pandemic stress: Associations with depression and anxiety in emerging adults in the U.S. Depression and Anxiety.

Kung, S., Alarcon, R. D., Williams, M. D., Poppe, K. A., Moore, M. J., \& Frye, M. A. (2013). Comparing the Beck depression inventoryII (BDI-II) and patient health questionnaire (PHQ-9) depression measures in an integrated mood disorders practice. Journal of Affective Disorders, 145, 341-343. https://doi.org/10.1016/j.jad. 2012.08.017

Linehan, M. M. (1993). Cognitive-behavioral treatment of borderline personality disorder. Guilford Press.

McGinty, E. E., Presskreischer, R., Anderson, K. E., Han, H., \& Barry, C. L. (2020). Psychological distress and COVID-19-related stressors reported in a longitudinal cohort of US adults in April and July 2020. Journal of the American Medical Association. Published online November 23, 2020. https://doi.org/10.1001/jama. 2020.21231

McKay, D., \& Asmundson, G. J. G. (2020). COVID-19 stress and substance use: Https://do current issues and future preparations. Journal of Anxiety Disorders, 74, 102274. https://doi.org/10.1016/j. janxdis.2020.102274
Millett, G. A., Jones, A. T., Benkeser, D., Baral, S., Mercer, L., Beyrer, C., Honermann, B., Lankiewicz, E., Mena, L., Crowley, J., Sherwood, J., \& Sullivan, P. S. (2020). Assessing differential impacts of COVID-19 on black communities. Annals of Epidemiology, 47, 37-44. https://doi.org/10.1016/j.annepidem. 2020.05.003

Nolen-Hoeksema, S., \& Aldao, A. (2011). Gender and age differences in emotion regulation strategies and their relationship to depressive symptoms. Personality and Individual Differences, 51(6), 704-708. https://doi.org/10.1016/j.paid.2011.06.012

Norton, P. J. (2007). Depression anxiety and stress scales (DASS-21): Psychometric analysis across four racial groups. Anxiety, Stress, and Coping, 20, 253-265. https://doi.org/10.1080/1061580070 1309279

Novacek, D. M., Hampton-Anderson, J. N., Ebor, M. T., Loeb, T. B., \& Wyatt, G. E. (2020). Mental health ramifications of the COVID19 pandemic for black Americans: Clinical and research recommendations. Psychological Trauma Theory Research Practice and Policy, 12(5), 449-451. https://doi.org/10.1037/tra0000796

Ogueji, I. A., Okoloba, M. M., \& Ceccaldi, B. M. D. (2021). Coping strategies of individuals in the United Kingdom during the COVID-19 pandemic. Current Psychology. https://doi.org/10. 1007/s12144-020-01318-7

Pulley, J. M., Jerome, R. N., Bernard, G. R., Olson, E. J., Tan, J., Wilkins, C. H., \& Harris, P. A. (2018). Connecting the public with clinical trial options: The ResearchMatch trials today tool. Journal of Clinical and Translational Science, 2, 253-257. https://doi.org/ $10.1017 /$ cts.2018.327

Rathod, S., Gega, L., Degnan, A., Pikard, J., Khan, T., Husain, N., Munshi, T., \& Naeem, F. (2018). The current status of culturally adapted mental health interventions: A practice-focused review of meta-analyses. Neuropsychiatric Disease and Treatment, 14, 165-178. https://doi.org/10.2147/NDT.S138430

Shamblaw, A. L., Rumas, R. L., \& Best, M. W. (2021). Coping during the COVID-19 pandemic: Relations with mental health and quality of life. Canadian Psychology, 62, 92-100. https://doi.org/10. 1037/cap0000263

Shanahan, L., Steinhoff, A., Bechtiger, L., Murray, A. L., Nivette, A., Hepp, U., Ribeaud, D., \& Eisner, M. (2020). Emotional distress in young adults during the COVID-19 pandemic: Evidence of risk and resilience from a longitudinal cohort study. Psychological Medicine, 1-10. https://doi.org/10.1017/S003329172000241X

Shechter, A., Diaz, F., Moise, N., Anstey, D. E., Ye, S., Agarwal, S., Birk, J. L., Brodie, D., Cannone, D. E., Chang, B., Claassen, J., Cornelius, T., Derby, L., Dong, M., Givens, R. C., Hochman, B., Homma, S., Kronish, I. M., Lee, S., ... Abdalla, M. (2020). Psychological distress, coping behaviors, and preferences for support among New York healthcare workers during the COVID-19 pandemic. General Hospital Psychiatry, 66, 1-8. https://doi.org/ 10.1016/j.genhosppsych.2020.06.007.

Taylor, S., Landry, C. A., Paluszek, M. M., Fergus, T. A., McKay, D., \& Asmundson, G. J. G. (2020). Development and initial validation of the COVID stress scales. Journal of Anxiety Disorders, 72, 1-7. https://doi.org/10.1016/j.janxdis.2020.102232

Tu, K. M., Erath, S. A., \& El-Sheikh, M. (2016). Coping responses moderate prospective associations between marital conflict and youth adjustment. Journal of Family Psychology, 30, 523-532. https://doi.org/10.1037/fam0000169

Vindegaard, N., \& Benros, M. E. (2020). COVID-19 pandemic and mental health consequences: Systemic review of current evidence. Brain, Behavior, and Immunity. https://doi.org/10.1016/j. bbi.2020.05.048

Wadsworth, M. E. (2015). Development of maladaptive coping: A functional adaptation to chronic, uncontrollable stress. Child Development Perspectives, 9(2), 96-100. https://doi.org/10.1111/ cdep. 12112 
Wadsworth, M. E., \& Compas, B. E. (2002). Coping with family conflict and economic strain: The adolescent perspective. Journal of Research on Adolescence, 12, 243-274. https://doi.org/10.1111/ 1532-7795.00033

Wang, D., Krase, K., MacMillan, T., Fishman, A. C., Witonsky, Y. R., \& Parris-Strigle, C. (2021). Micro, mezzo and macro factors associated with coping in the early phase of COVID-19. Journal of Human Behavior in the Social Environment, 31, 1-4. https:// doi.org/10.1080/10911359.2020.1838985

Weiss, N. H., Johnson, C. D., Contractor, A., Peasant, C., Sawn, S. C., \& Sullivan, T. P. (2017). Racial/ethnic differences moderate associations of coping strategies and posttraumatic stress disorder symptom clusters among women experiencing partner violence: A multigroup path analysis. Anxiety, Stress, and Coping, 30, 347 363. https://doi.org/10.1080/10615806.2016.1228900

Weisz, J. R., McCabe, M. A., \& Dennig, M. D. (1994). Primary and secondary control among children undergoing medical procedures: Adjustment as a function of coping style. Journal of
Consulting and Clinical Psychology, 62, 324-332. https://doi. org/10.1037/0022-006X.62.2.324

World Health Organization. (2021, March 30). WHO Coronavirus Disease (COVID-19) Dashboard. https://covid19.who.int/

Xiong, J., Lopsitz, O., Nasri, F., Lui, L. M. W., Gill, H., Phan, L., Chen-Li, D., Iacobucci, M., Ho, R., Majeed, A., McIntyre, R. S., et al. (2020). Impact of COVID_19 pandemic on mental health in the general population: A systematic review. Journal of Affective Disorders, 277, 55-64. https://doi.org/10.1016/j.jad.2020.08.001 Yancy, C. W. (2020). COVID-19 and African Americans. JAMA, 323(19), 1891-1892. https://doi.org/10.1001/jama.2020.6548

Publisher's Note Springer Nature remains neutral with regard to jurisdictional claims in published maps and institutional affiliations. 